\title{
3D Reconstruction from Tangent-of-Sight Measurements of a Moving Object Seen from a Moving Camera
}

\author{
Dana Segal and Amnon Shashua \\ School of Computer Science and Engineering, \\ The Hebrew University, \\ Jerusalem 91904, Israel \\ e-mail: \{danna,shashua\}@cs.huji.ac.il
}

\begin{abstract}
Consider the situation of a monocular image sequence with known ego-motion observing a $3 D$ point moving simultaneously but along a path of up to second order, i.e. it can trace a line in $3 D$ or a conic shaped path. We wish to reconstruct the $3 D$ path from the projection of the tangent to the path at each time instance. This problem is analogue to the "trajectory triangulation" of lines and conic sections recently introduced in [1,3], but instead of observing a point projection we observe a tangent projection and thus obtain a far simpler solution to the problem.

We show that the $3 D$ path can be solved in a natural manner, and linearly, using degenerate quadric envelopes - specifically the disk quadric. Our approach works seamlessly with both linear and second order paths, thus there is no need to know in advance the shape of the path as with the previous approaches for which lines and conics were treated as distinct. Our approach is linear in both straight line and conic paths, unlike the non-linear solution associated with point trajectory [3].

We provide experiments that show that our method behaves extremely well on a wide variety of scenarios, including those with multiple moving objects along lines and conic shaped paths.
\end{abstract}

\section{Introduction}

There has been a recent drive towards extending the envelope of multi-view $3 \mathrm{D}$ reconstruction and Image-based Rendering beyond the static scene assumption in the sense of considering "dynamic" situations in which a point to be reconstructed, or view-morphed, is moving simultaneously while the camera is in motion $[1,3,2]$. In these situations only line-of-sight measurements are available, that is, optical rays from distinct camera positions do not intersect (triangulate) at a point in space (as in the conventional multi-view reconstruction paradigm), thus new techniques need to be introduced in order to handle these cases. This paradigm was named "trajectory triangulation" by $[1,3]$. 
In this paper we consider a related problem defined as follows. Consider a point $P$ moving in space along a path $\gamma$ which could either be a straight line or a conic in $3 \mathrm{D}$, i.e., a path up to second order in space coordinates. The path $\gamma$ is observed by a generally moving camera whose ego-motion (camera projection matrices) is known. The observation takes the form of the projections of tangents to the curve $\gamma$. In other words, in each image we see one (or more) tangents of $\gamma$ as a line in the image plane (we refer to this as "tangent-of-sight" measurement) and our task is to reconstruct $\gamma$ from the image measurements across multiple (at least 2) views. See Fig. 1 for illustration of the problem.

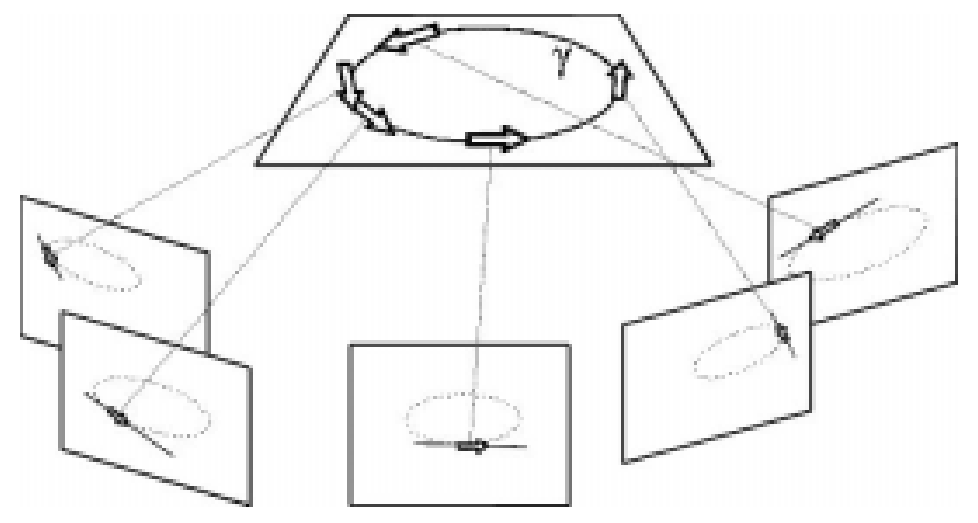

Fig. 1. The conic/line path $\gamma$ is observed by a generally moving camera where each image views the projection of a local tangent to the curve. The task is to reconstruct $\gamma$ from such "tangent-of-sight" measurements across a sequence of views.

Note that in our problem an observation is a line segment (projection of the tangent to $\gamma$ in space), where as in $[1,3]$ the observation is an image point corresponding to a moving point $P$ along $\gamma$. This change in problem definition makes a significant difference in the kind of tools one can use for the solution. In the case of a point observation it was shown that when $\gamma$ is a straight line the solution (Plucker coordinates of the line) is linear in the image observations and 5 views are necessary - whereas when $\gamma$ is a conic 9 views are necessary and the solution is non-linear. Moreover, the solution assuming $\gamma$ is a conic would break-down if $\gamma$ is a straight line (and will suffer from numerical instability in case $\gamma$ is close to a straight line).

We will show that in the case of tangent-of-sight observations a natural tool for approaching the reconstruction of $\gamma$ is the degenerate quadric envelope - in particular the disk quadric. Quadric envelopes were introduced in the computer vision literature in the context of camera self-calibration (the "absolute quadric" [4]), but here we make a different use of them. In our approach, each observation contributes a linear equation for a disk quadric, thus in the case each image contains only one observation one would need at least 9 views (or 8 views for a 
4 -fold ambiguity). Given the disk quadric it becomes a simple matter to extract from it the parameters of $\gamma$ ( 3 parameters of the plane and 5 parameters of the conic on the plane). Moreover, the technique would work seamlessly without changes when $\gamma$ is a straight line as well.

On a practical level, measurements of line segments are quite naturally obtained from images. Moreover, the occluding contour of a moving object would provide the tangent-of-sight measurement necessary for our computations. Thus, the introduction of tangent-of-sight observations in the realm "trajectory triangulation" is both practical and feasible.

\section{Background: Cameras and Quadric Loci and Envelopes}

We will be working with the projective $3 \mathrm{D}$ space and the projective plane. In this section we will describe the basic elements we will be working with: (i) camera projection matrices, and (ii) Quadric envelopes and the disk quadric.

A point in the projective plane $\mathcal{P}^{2}$ is defined by three numbers, not all zero, that form a coordinate vector defined up to a scale factor. The dual projective plane represents the space of lines which are also defined by a triplet of numbers. A point in the projective space $\mathcal{P}^{3}$ is defined by four numbers, not all zero, that form a coordinate vector defined up to a scale factor. The dual projective space $\mathcal{P}^{*^{3}}$ represents the space of planes which are also defined by a quadruple of numbers.

The projection from $3 \mathrm{D}$ space to $2 \mathrm{D}$ space is determined by a $3 \times 4$ matrix. If $P, p$ are corresponding $3 \mathrm{D}$ and $2 \mathrm{D}$ points, then $p \cong M P$, where $\cong$ denotes equality up to scale. If $\lambda$ is a line in $2 \mathrm{D}$, then $\lambda^{\top} M$ is the plane passing through the line $\lambda$ and the projection center of the camera. The plane is referred to as the "visual plane" of $\lambda$.

A quadric locus is a second order polynomial of the $3 \mathrm{D}$ projective coordinates representing points on a quadric surface. The points $P \in \mathcal{P}^{3}$ satisfying the equation $P^{\top} Q P=0$, where $Q$ is a symmetrical $4 \times 4$ matrix define a quadric locus. If $P$ is on the quadric surface, then $Q P$ is the tangent plane at that point.

A quadric envelope is a second order polynomial of the $3 \mathrm{D}$ coordinates of the dual space - the space of planes. The planes $U \in \mathcal{P}^{*^{3}}$ that satisfy the equation $U^{\top} Q^{*} U=0$, where $Q^{*}$ is a symmetric matrix, are tangents of a quadric surface. If $U$ is a tangent to the surface (belongs to the envelope), then $Q^{*} U$ is the point on the surface defined by the intersection of the tangent plane and the surface. If $Q$ is full rank, then by the principle of point-plane duality we have

$$
0=(Q P)^{\top} Q^{*}(Q P)=P^{\top}\left(Q^{\top} Q^{*} Q\right) P \quad \Longrightarrow Q^{*}=Q^{-T}
$$

In other words, one can move from point-equation to plane-equation of a quadric simply by taking the cofactors matrix, i.e., $Q^{*}$ can be described by the cofactors of $Q$ (and vice versa).

A full rank quadric (locus and envelope) is called a proper quadric. A rank-3 quadric is called a quadric cone. Let $Q \mathcal{X}=0$, then clearly the point $\mathcal{X}$ belongs 
to the surface because $\mathcal{X}^{\top} Q \mathcal{X}=0$ as well. Let $P$ be any other point on the surface, i.e., $P^{\top} Q P=0$. Then, the entire line $\alpha P+\beta \mathcal{X}$ is also on the surface:

$$
(\alpha P+\beta \mathcal{X})^{\top} Q(\alpha P+\beta \mathcal{X})=0 \quad \forall \alpha, \beta
$$

Therefore, the quadric cone is generated by lines through the point (apex) $\mathcal{X}$. The co-factor matrix $Q^{*}$ is a rank-1 matrix as follows. All the planes through $\mathcal{X}$ are tangent planes, i.e., $U^{\top} Q^{*} U=0$ and $U^{\top} \mathcal{X}=0$, i.e., the rows of $Q^{*}$ are scaled versions of $\mathcal{X}$, and $U^{\top} Q^{*} U=0$ is is the plane equation of $\mathcal{X}$ taken twice (repeated plane).

A rank-3 quadric envelope is called a disk quadric. Let $Q^{*} \mathcal{Y}=0$, then $\mathcal{Y}$ is tangent to the surface because $\mathcal{Y}^{\top} Q^{*} \mathcal{Y}=0$ as well. If $U$ belongs to the envelope, i.e., $U^{\top} Q^{*} U=0$, then the pencil of planes $\alpha U+\beta \mathcal{Y}$ belong to the envelope as well. Therefore, the disk quadric is a "disk" of coplanar points where the boundary of the disk is a conic section, that is, the envelope includes all the planes through all the tangents to the boundary conic, i.e., $\infty^{1}$ pencils of $\infty^{1}$ planes (see Fig. 2). The matrix of co-factors is a rank-1 matrix whose rows are scaled versions of $\mathcal{Y}$, the plane of the disk.

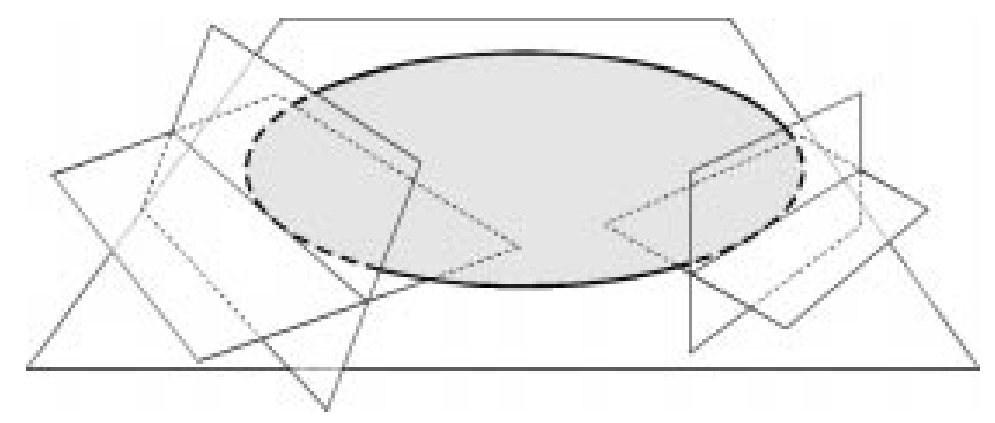

Fig. 2. A disk quadric is a "disk" of coplanar points where the boundary of the disk is a conic section, that is, the envelope includes all the planes through all the tangents to the boundary conic, i.e., $\infty^{1}$ pencils of $\infty^{1}$ planes.

It is important to note that the application of the principle of duality (matrix of cofactors) does not hold for rank-deficient quadrics. The disk quadric is the dual of the proper cone, yet the transformation between point-equation and plane-equation cannot be achieved solely through the matrix of cofactors. We will return to this issue later in the paper.

Finally, a rank-2 quadric locus describes a pair of distinct planes, and a rank2 quadric envelope describes a pair of distinct points. Table 1 summarizes the rank classifications of quadric loci and quadric envelopes. 


\begin{tabular}{||l|l|l||}
\hline$\rho$ & Quadric Locus & Quadric Envelope \\
\hline 4 & Proper Quadric & Proper Quadric Envelope \\
\hline 3 & Proper Cone & Disk Quadric \\
\hline 2 & Pair of planes (line) & Pair of points(line) \\
\hline 1 & Repeated Plane & Repeated Point \\
\hline
\end{tabular}

Table 1. Classification of quadrics according to rank

\section{Reconstruction of a Conic Path from Tangents-of-Sight}

We wish to recover the path of an object moving in space from a sequence of images without prior knowledge of the shape of the path (line in 3D or planar conic in 3D). We will present a method for linearly recovering the path from 9 views, given their 9 projection matrices and a tangent to the path of motion in each view. The output of the algorithm in the case of a planar conic will be the 3 parameters of the plane and the 5 parameters of the conic on that plane. In case of a line the output will be the Plucker coordinates of the line in 3D. Our method is based on the fact that both a planar conic in 3D and a line in 3D have a representation as a degenerate quadric envelope.

Our problem can be stated as follows. We are given tangents of a moving point (or equivalently we are observing a moving tangent line tracing a conic envelope in space) across a number of views seen from a monocular sequence with known ego-motion, i.e., the camera projection matrices are assumed to be known. We wish to recover the conic path and to reconstruct the $3 \mathrm{D}$ position of the moving point at each time instance. See Fig. 1.

Given the background on quadric envelopes, it becomes clear that the conic trajectory is part of a disk quadric. The observations we obtain from the image space is the projection of a pencil of planes (a tangent line to the boundary of the disk quadric) at each time instance.

Let $l_{i}$ be the tangent line measured in view $i$, and let $M_{i}$ be the camera projection matrix at time $i$. Then $l_{i}^{\top} M_{i}$ is the visual plane which is tangent to the disk quadric. We have therefore the linear set of equations:

$$
l^{\top} M_{i} Q^{*} M_{i}^{\top} l_{i}=0
$$

which provides a unique solution for $Q^{*}$ using 9 views, or a 4 -fold ambiguity using 8 views (because we know that the determinant of $Q^{*}$ must vanish). It is a reasonable assumption that if the moving camera is viewing a single moving object we would be able to extract one tangent to the path of motion per image, tracking some visible feature on the object, but we are not restricted to using one tangent in each view. Indeed, if there appear to be multiple tangents to the same path of motion, for example, when tracking a train consisting of many cars moving along it's track (as shown in Fig. 3) more than one tangent can be used per image. The 9 visual planes can be acquired along less than 9 images as well. The only limitation on the number of images is that the sequence must be at least 2 images long. 
Given that we have found the disk quadric $Q^{*}$, the reconstructed $3 \mathrm{D}$ points are simply $P_{i} \cong Q^{*} M_{i}^{\top} l_{i}$. The plane $\pi$ on which the conic resides is the null space of $Q^{*}$, i.e., $Q^{*} \pi=0$. To recover the point-equation of the conic path we do the following.

We have seen previously that with rank-deficient quadrics the cofactors are not sufficient for moving between plane-equation and point-equation form. We describe the conic as the intersection of a proper cone and the plane $\pi$. The apex of the proper cone can be chosen arbitrarily, thus let $\mathcal{X}$ be a the chosen apex of the cone we wish to construct. $U^{\top} Q^{*} U=0$ describe the plane-equation of the disk quadric and include all tangent planes to the conic we wish to recover, thus by adding the constraint $U^{\top} \mathcal{X}=0$ we obtain a subset of the tangents planes that define the plane-equation of the enveloping cone whose apex is at $\mathcal{X}$. For every such tangent plane $U, P=Q^{*} U$ is a point on the conic section. We wish to express $U$ as a function (uniquely) of point coordinates $P$, as follows. We have two sources of equations: $Q^{*} U=P$ and $\mathcal{X}^{\top} U=0$, i.e.,

$$
\left[\begin{array}{l}
Q^{*} \\
\mathcal{X}^{\top}
\end{array}\right] U=\left(\begin{array}{c}
P \\
0
\end{array}\right)
$$

and from the pseudo-inverse we obtain:

$$
U=\left(Q^{*} Q^{*}+\mathcal{X} \mathcal{X}^{\top}\right)^{-1} Q^{*} P=Q^{\#} P
$$

Note that the matrix in parenthesis is of full rank because of the addition of $\mathcal{X} \mathcal{X}^{\top}$. Substituting $U$ in the plane-equation $U^{\top} Q^{*} U=0$ we obtain the pointequation:

$$
P^{\top} Q^{\#^{\top}} Q^{*} Q^{\#} P=0
$$

i.e., the rank-3 quadric locus (quadric cone) is $Q=Q^{\#^{\top}} Q^{*} Q^{\#}$. A numerical example of transformation between plane and point equations for degenerate quadrics would be helpful.

Suppose we are given the plane equation of a disk quadric:

$$
\begin{aligned}
& U_{1}^{2}+4 U_{1} U_{2}+6 U_{1} U_{3}-6 U_{1} U_{4}+U_{2}^{2}+ \\
& U_{3}^{2}-2 U_{3} U_{4}+U_{4}^{2}=0
\end{aligned}
$$

Or in matrix representation:

$$
Q^{*}=\left[\begin{array}{rrrr}
1 & 2 & 3 & -3 \\
2 & 1 & 0 & 0 \\
3 & 0 & 1 & -1 \\
-3 & 0 & -1 & 1
\end{array}\right]
$$

The plane of the conic is given by the null space of $Q^{*}$ and is therefore the plane $[0,0,1,1]$, or $Z+1=0$. We now define the cone $K^{*}$ of enveloping planes tangent to $Q^{*}$ that coincide with the point $\mathcal{X}=[0,0,0,1]^{T}$, by setting $U_{4}=0$ in Eq. 1. We get the plane equation of $K^{*}$ :

$$
U_{1}^{2}+4 U_{1} U_{2}+6 U_{1} U_{3}+U_{2}^{2}+U_{3}^{2}=0
$$


Or in matrix representation:

$$
Q^{*}=\left[\begin{array}{llll}
1 & 2 & 3 & 0 \\
2 & 1 & 0 & 0 \\
3 & 0 & 1 & 0 \\
0 & 0 & 0 & 0
\end{array}\right]
$$

Next, we translate the plane equation of the cone into its point equation, by expressing it in the coordinates of the tangency point to a general plane $U \in K^{*}$ :

$$
K^{*} U=\left[\begin{array}{c}
U_{1}+2 U_{2}+3 U_{3} \\
2 U_{1}+U_{2} \\
3 U_{1}+U_{3} \\
0
\end{array}\right]=\left(\begin{array}{c}
X \\
Y \\
Z \\
W
\end{array}\right)
$$

We solve Eq. 5 for $U_{1}, U_{2}, U_{3}$ thus, expressing them as linear combinations of $X, Y, Z$ :

$$
\begin{aligned}
& U_{1}=\frac{-1}{12} X+\frac{1}{6} Y+\frac{1}{4} Z \\
& U_{2}=\frac{2}{3} Y+\frac{1}{6} X-\frac{1}{2} Z \\
& U_{3}=\frac{1}{4} Z+\frac{1}{4} X-\frac{1}{2} Y
\end{aligned}
$$

Substituting Eq. 6 for $U_{1}, U_{2}, U_{3}$ in the plane equation, gives us the point equation of the cone $K$, in $X, Y, Z$ :

$$
\begin{aligned}
& X^{2}-4 X Y-6 X Z-8 Y^{2}+ \\
& 12 Y Z-3 Z^{2}=0
\end{aligned}
$$

We now have the point equations of two surfaces, defining the conic in terms of points in space.

The intersection of these two surfaces is the conic on the plane, the collection of points which belong both to the cone and to the plane of the disk quadric. The equation of the plane gives us $Z=-1$, which we substitute in Eq. 7 and we get the equation of the conic on the plane:

$$
X^{2}-4 X Y+6 X-8 Y^{2}-12 Y-3=0
$$

\subsection{Recovering Plucker Coordinates of a Line}

Given a quadric envelope $Q^{*}$ representing a line in $3 \mathrm{D}$, we would like to recover the Plucker coordinates of the line. As we learn from Table $1, Q^{*}$ is of rank 2 and represents a line by encoding the information of a pair of $3 \mathrm{D}$ points that coincide with the line. The null space of $Q^{*}$ is of dimension 2 and consists of two planes:

$$
\operatorname{null}\left(Q^{*}\right)=\left[\pi_{1}, \pi_{2}\right]
$$

These planes satisfy $\pi_{1} Q^{*} \pi_{1}^{T}=0, \pi_{2} Q^{*} \pi_{2}^{T}=0$, thus they are tangent to $Q^{*}$. Since $Q^{*}$ is a line, it must lie on both planes $\pi_{1}$ and $\pi_{2}$, it is the line of intersection 
of $\pi_{1}$ and $\pi_{2}$. We can find two points $P, Q$ on the line of intersection and perform the join operation on them to get the Plucker coordinates of the line.

$$
\begin{aligned}
& {[P, Q]=\text { null }\left(\begin{array}{c}
\pi_{1} \\
\pi_{2}
\end{array}\right) } \\
& L=P \wedge Q \\
&= {\left[X_{p}-X_{q}, Y_{p}-Y_{q}, Z_{p}-Z_{q},\right.} \\
& X_{p} Y_{q}-Y_{p} X_{q}, X_{p} Z_{q}-Z_{p} X_{q}, \\
&\left.Y_{p} Z_{q}-Z_{p} Y_{q}\right]
\end{aligned}
$$

\section{Experiments}

We have conducted a number of experiments on both synthetic and real image sequences. We report here a number of examples of real image sequences, varying in the amount and type of measurements used for recovering the motion path $\gamma$ of a moving object. For each experiment we will present the input sequence (in whole or partially) showing the tangent-of-sight lines used as input and the resulting images, which show the recovered path $\gamma$.

In all the examples, we used sequences taken by a hand-held moving camera. The tangents in the images were marked manually and the projection matrices were recovered from matching points in the static background - both the tangents and the projection matrices were passed to the algorithm as input.

The first example demonstrates the unifying quality of the algorithm, handling a scene containing both a conic trajectory and a line trajectory. We took a sequence of 9 images viewing a toy train moving along its circular track and a toy jeep moving along a straight line. Both for the trajectory of the train and for the trajectory of the car, one tangent was used in each of the 9 images. The tangents to the trajectory of the train were taken from the bottom of the engine car. The tangents to the trajectory of the jeep were taken where the wheels touch the chess-board. Fig. 3 shows the 9 input images, in each image the tangent to the train is drawn in black and the tangent to the jeep is drawn in white

Fig. 4 shows the result of this experiment. The images shown are a subset of the image sequence. In each image the recovered conic trajectory of the train, projected to the image, is drawn in black and the recovered line trajectory of the jeep is drawn in white.

The second example was designed for quantitative estimation of accuracy. The example was created using 9 images viewing a spinning turntable, with one tangent to the turntable taken in each image. In the background we placed a static object, used for Euclidean calibration, consisting of three planes orthogonal to each other featuring a regular chess-board pattern. The plane on the floor was taken to be $Y=100$ and the two other planes were taken to be $X=0$ and $Z=0$. The projection matrices were created from points on the calibration object. The size of each square on the grid is $2.5 \times 2.5 \mathrm{~cm}$, and was taken to be $10 \times 10 \mathrm{in}$ out coordinate system. The height of the turntable is $3 \mathrm{~cm}$, the plane of the turntable 

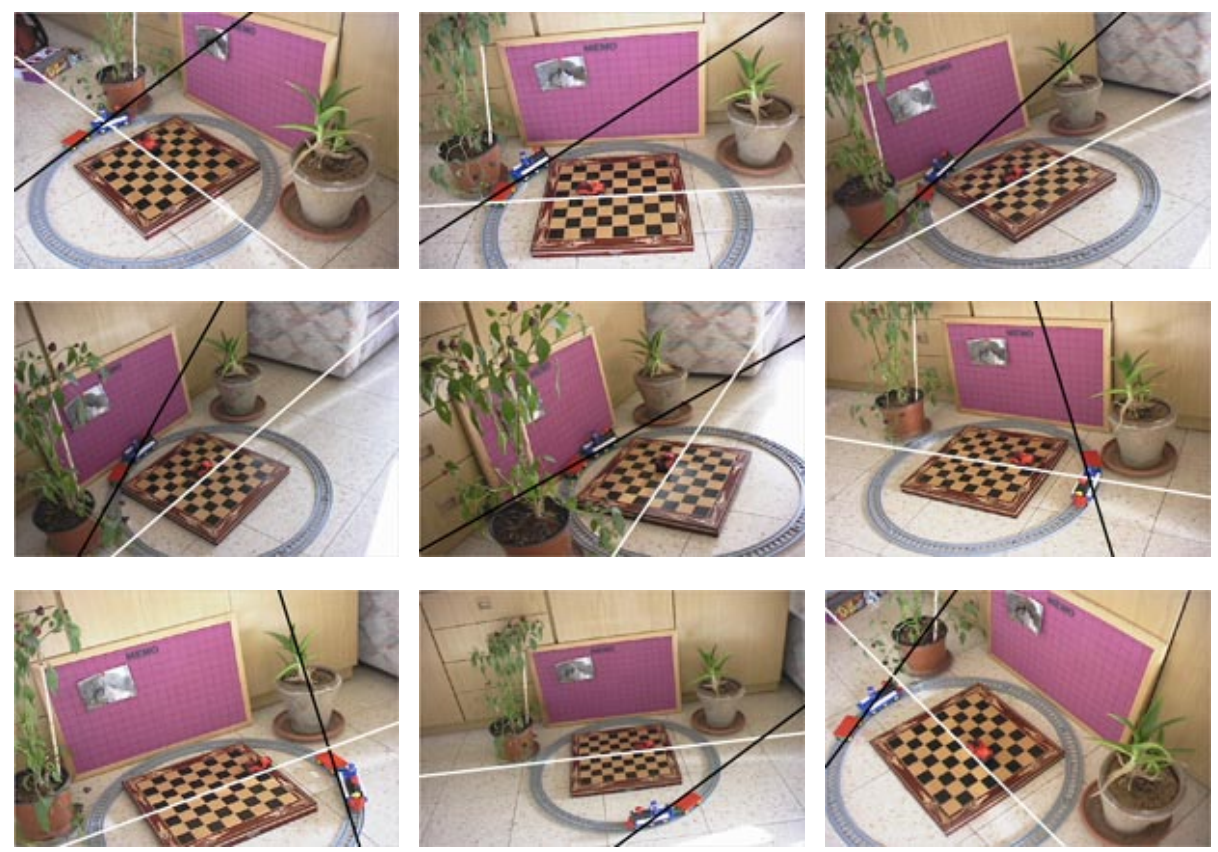

Fig. 3. Input sequence for 1st example, 9 images containing one tangent to each trajectory per image. Tangents to conic trajectory are drawn in black, tangents to line trajectory are drawn in white.

is thus the plane $Y=120$. The plane of the conic recovered by the algorithm was:

$$
0.0026 X+Y+0.0292 Z=112.1446
$$

which is very close to the known plane. Fig. 5 shows 4 of the input images used in this example with the tangent taken in each image, the projection of the conic onto each image and a regular grid on the plane of the conic.

\section{$5 \quad$ Summary}

We have introduced the problem of recovering the path of a moving object seen from a moving camera using tangent-of-sight measurements and have shown that the degenerate quadric envelope is a natural tool for the problem of reconstructing second-order paths in space.

Our technique is very simple as each observation provides a linear estimate to a disk quadric that contains all the necessary information for recovering the 8 parameters of the conic shaped path. A byproduct of our approach is that the system does not breakdown when the the path is a straight line as it is a special case of a degenerate quadric envelope. 

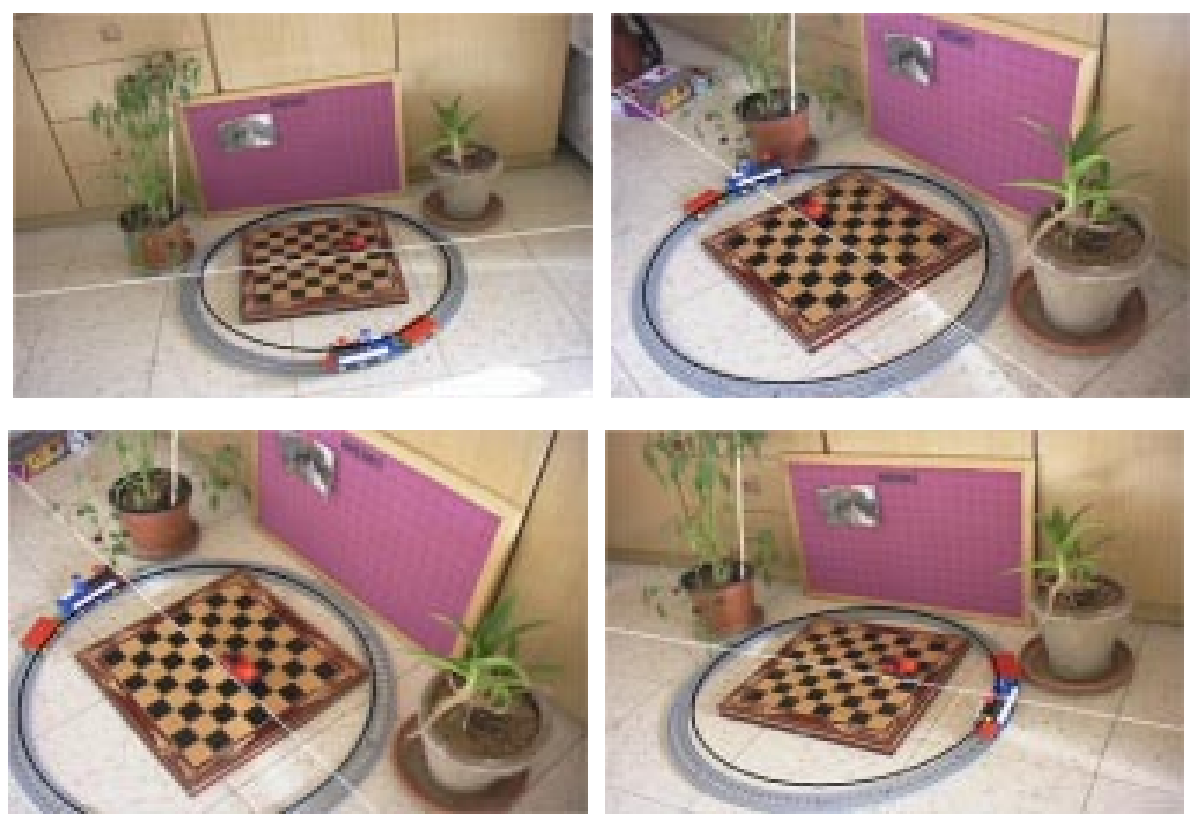

Fig. 4. Recovered planar conic (in black) and line (in white) are projected to several reference images from the input sequence

\section{References}

1. S. Avidan and A. Shashua. Trajectory triangulation of lines: Reconstruction of a 3d point moving along a line from a monocular image sequence. In Proceedings of the IEEE Conference on Computer Vision and Pattern Recognition, Jume 1999.

2. R.A. Manning and C.R. Dyer. Interpolating view and scene motion by dynamic view morphing. In Proceedings of the IEEE Conference on Computer Vision and Pattern Recognition, pages 388-394, Fort Collins, Co., June 1999.

3. A. Shashua, S. Avidan, and M. Werman. Trajectory triangulation over conic sections. In Proceedings of the International Conference on Computer Vision, pages 330-336, Corfu, Greece, September 1999.

4. B. Triggs. Autocalibration and the absolute quadric. In Proceedings of the IEEE Conference on Computer Vision and Pattern Recognition, pages 609-614, Puerto Rico, June 1997. IEEE Computer Society Press. 

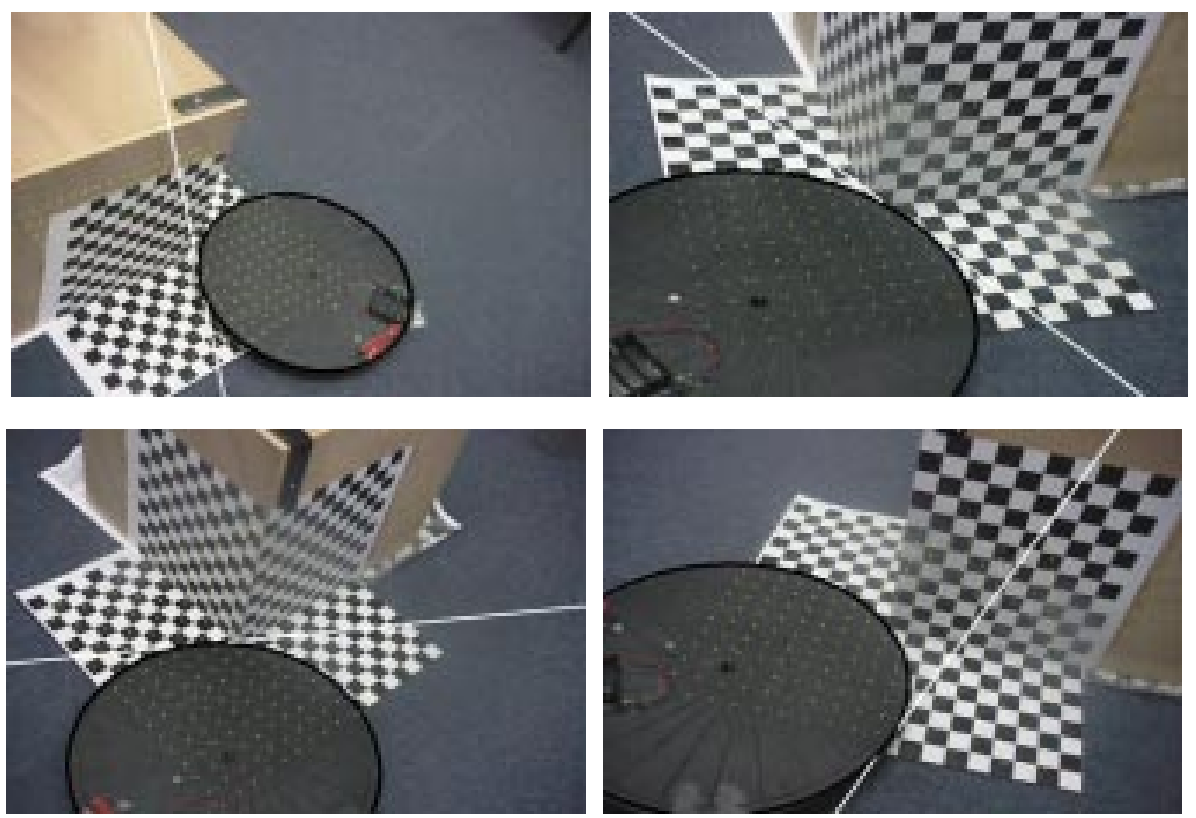

Fig. 5. 4 out of 9 images from input sequence used for numerical example. Images show projection of the conic to each image drawn in black, tangent in each image drawn in white and a regular grid of bright dots on the plane of the conic. 